\title{
Nucleon-Nucleon Scattering in a Strong External Magnetic Field and the Neutrino Emissivity
}

\author{
E. Bavarsad ${ }^{a}$, M. Haghighat ${ }^{a, b}$ and R. Mohammadi ${ }^{a}$ \\ a) Department of Physics, Isfahan University of Technology, \\ Isfahan 84156-83111, Iran \\ b) Department of Physics, University of Alabama, \\ Tuscaloosa, Alabama 35487, USA
}

\begin{abstract}
The nucleon-nucleon scattering in a large magnetic background is considered to find its potential to change the neutrino emissivity of the neutron stars. For this purpose we consider the one-pion-exchange approximation to find the NN cross-section in a background field as large as $10^{15} \mathrm{G}-10^{18} \mathrm{G}$. We show that the NN cross-section in neutron stars with temperatures in the range 0.1-5 MeV can be changed up to the one order of magnitude with respect to the one in the absence of the magnetic field. In the limit of the soft neutrino emission the neutrino emissivity can be written in terms of the NN scattering amplitude therefore the large magnetic fields can dramatically change the neutrino emissivity of the neutron stars as well.
\end{abstract}




\section{Introduction}

Neutron stars in general can have magnetic fields as large as $10^{12} \mathrm{G}-10^{18} \mathrm{G}$ [1], [2], [3], [4], [5], 6] while the timing observations indicate that the surface magnetic fields of the radio pulsars are about $10^{12} \mathrm{G}$ [7], by equating magnetic field energy to the gravitational binding energy, one can estimate an upper limit for strength of the magnetic field to be $B \sim 10^{18} \mathrm{G}[$ ]. Meanwhile cooling processes in the neutron stars formation that has long been considered by many physicists, can be affected by such a large magnetic field in two ways. It can change the electron or the photon conductivities [9], [2], [10] as well as the wave functions and the propagators of the particles involving in the cooling processes. One of the most important reactions during the thermal evolution of the neutron stars is the emission of neutrino via the bremsstrahlung process

$$
N N \rightarrow N N+\nu \bar{\nu}
$$

In the first sight it seems that since the mass of the nucleons are about $1 \mathrm{Gev}$ therefore the magnetic fields even as large as $10^{18} \mathrm{G}$ can not significantly change the cross section. However one should note that there are more energy scales besides the mass of the nucleons. In fact one of the scales is the temperature of the evolving neutron star which becomes less than $1 \mathrm{MeV}$ in a few seconds of the late-time cooling phase and is comparable with $B \sim 10^{13} \mathrm{G}$. Furthermore if one consider one-pion-exchange (OPE) for the nucleon-nucleon interaction then the magnetic fields of the order of $10^{17} \mathrm{G}$ and more can change the strong coupling constant as well. Meanwhile in such a low temperature era the bremsstrahlung process can only produce the soft neutrinos with respect to the initial energies and therefore the neutrino emissivity can be related to the on-shell NN scattering amplitude [11]. Thus to find the neutrino emissivity one can study the nucleon-nucleon scattering separately. The amplitude can be even constructed from NN scattering data, as is done in [11] as a model independent way to describe the neutrino emissivity. But the usual studies on the nucleon-nucleon scattering in OPE approximation [12] and more precisely the existing data are in the absence of the magnetic field. Our aim in this paper is to examine the magnetic field dependence of the nucleon-nucleon scattering in the OPE approximation when soft neutrino approximation is applicable.

The paper is organized as follows: in section 2 we give the field operators of the proton and neutrons in an external magnetic field. Consequently we obtain the cross section of the scattering in the magnetic field in section 3. An estimation for the effect of strong magnetic field on the neutrino emissivity is presented in section 4 . In section 5 
we give some concluding remarks. Appendix A is devoted to solving the Dirac equation for proton and neutron in an external magnetic field. In Appendix B we present a simplified expression for the amplitude matrix elements of the scattering process.

\section{Fermion field operator}

To study the proton-neutron scattering in a strong external magnetic field, the field operators of the proton and neutron are needed. Since the field operators contain wave functions and energy spectrum, Dirac equation in presence of an external magnetic field must be solved. We choose the external magnetic field to be in the positive $z$-axis $\vec{B}=B \hat{k}$. Because of gauge invariance there is a freedom in the choice of vector potential $A_{\mu}(x)$, thus for simplicity we choose

$$
A_{\mu}(x)=(0, y B, 0,0)
$$

This choice of vector potential pushes all the $y$-dependence into the spinors alone and not in the phases, so it is useful to introduce the following notations

$$
\begin{aligned}
x_{y}^{\mu} & =(t, x, 0, z), \\
\vec{V}_{y} & =\left(V_{x}, V_{z}\right),
\end{aligned}
$$

where $\vec{V}_{y}$ is any 2-vector. The solutions of the Dirac equation for proton and neutron in the external magnetic field (2) are presented in the Appendix A. By using this solutions the proton field operator can be written as

$$
\psi_{p}(x)=\sum_{n=0}^{\infty} \sum_{s= \pm 1} \int \frac{d^{2} \vec{p}_{y}}{(2 \pi)^{2}} N_{s}\left[a_{s}\left(n, \vec{p}_{y}\right) U_{s}\left(y, n, \vec{p}_{y}\right) e^{-i p \cdot x_{y}}+b_{s}^{\dagger}\left(n, \vec{p}_{y}\right) V_{s}\left(y, n, \vec{p}_{y}\right) e^{+i p . x_{y}}\right],
$$

where the creation and annihilation operators obey the following anticommutation relations

$$
\left\{a_{s}\left(n, \vec{p}_{y}\right), a_{s^{\prime}}^{\dagger}\left(n^{\prime}, \vec{p}_{y}^{\prime}\right)\right\}=\left\{b_{s}\left(n, \vec{p}_{y}\right), b_{s^{\prime}}^{\dagger}\left(n^{\prime}, \vec{p}_{y}^{\prime}\right)\right\}=(2 \pi)^{2} \delta_{s, s^{\prime}} \delta_{n, n^{\prime}} \delta_{y}^{2}\left(\vec{p}^{\prime}-\vec{p}\right) .
$$

With the help of (5), the explicit form of the wave functions given in the equations (61), (64) and the completeness relation (53), it can be shown that the equal-time anticommutation relation is satisfied

$$
\left\{\psi_{p}(t, \vec{x}), \psi_{p}^{\dagger}\left(t, \vec{x}^{\prime}\right)\right\}=\delta^{3}\left(\vec{x}-\vec{x}^{\prime}\right)
$$


For convenience we normalize our 1-particle states in a box with dimensions $L_{x} L_{y} L_{z}=V$ such that the proton state is

$$
\left|p\left(n, \vec{p}_{y}, s\right)>=\frac{1}{\sqrt{L_{x} L_{z}}} a_{s}^{\dagger}\left(n, \vec{p}_{y}\right)\right| 0>.
$$

Similarly for the neutron field operator one has

$$
\psi_{n}(x)=\sum_{s= \pm 1} \int \frac{d^{3} \vec{p}}{(2 \pi)^{3}} N_{s}\left[a_{s}(\vec{p}) U_{s}(\vec{p}) e^{-i p \cdot x}+b_{s}^{\dagger}(\vec{p}) V_{s}(\vec{p}) e^{+i p . x}\right],
$$

in which the creation and annihilation operators obey the following anticommutation relations

$$
\left\{a_{s}(\vec{p}), a_{s^{\prime}}^{\dagger}\left(\vec{p}^{\prime}\right)\right\}=\left\{b_{s}(\vec{p}), b_{s^{\prime}}^{\dagger}\left(\vec{p}^{\prime}\right)\right\}=(2 \pi)^{3} \delta_{s, s^{\prime}} \delta^{3}\left(\vec{p}^{\prime}-\vec{p}\right) .
$$

By using the relations (9) and the explicit form of the wave functions given in the equations (66) and (69) it is easy to show that equal-time anticommutation relation is satisfied

$$
\left\{\psi_{n}(t, \vec{x}), \psi_{n}^{\dagger}\left(t, \vec{x}^{\prime}\right)\right\}=\delta^{3}\left(\vec{x}-\vec{x}^{\prime}\right) .
$$

Box normalization condition for the neutron 1-particle state is

$$
\left|n(\vec{p}, s)>=\frac{1}{\sqrt{V}} a_{s}^{\dagger}(\vec{p})\right| 0>.
$$

\section{Cross section of the scattering in the external magnetic field}

Our aim in this section is to explore the scattering cross section of $p+n \rightarrow p+n$ in the background of a strong magnetic field, $\sigma_{B}$, using the one-pion-exchange approximation. The deferential cross section can be written as

$$
d \sigma=\frac{V}{\left|\vec{v}_{p}-\vec{v}_{n}\right|} \frac{\overline{|S|^{2}}}{T} d \rho
$$

where $\left|\vec{v}_{p}-\vec{v}_{n}\right| / V$ is the flux of the incident particles, $T$ is the time interval, $\overline{|S|^{2}}$ is the average of the squared scattering matrix elements and $d \rho$ is the deferential phase space of the final states. The effective low energy Lagrangian for pion-nucleon interaction is

$$
\mathcal{L}_{i n t}=g_{\pi N}\left[\bar{\psi}_{p} \gamma^{5} \psi_{p} \phi_{\pi^{0}}+\sqrt{2} \bar{\psi}_{n} \gamma^{5} \psi_{p} \phi_{\pi^{-}}+\sqrt{2} \bar{\psi}_{p} \gamma^{5} \psi_{n} \phi_{\pi^{+}}-\bar{\psi}_{n} \gamma^{5} \psi_{n} \phi_{\pi^{0}}\right]
$$

where $g_{\pi N} \simeq 14$ is the pion-nucleon coupling constant and $\psi_{n}, \psi_{p}$ and $\phi$ are neutron, proton and pion fields, respectively. To obtain the scattering matrix elements in the 
external magnetic field we use the standard method where the wave functions and propagators are modified with the external magnetic field while the vertices are leaved unchanged. The proton-neutron scattering at the tree level involves two diagrams, one is mediated by the neutral pion $S_{n c}$

$$
\begin{array}{r}
S_{n c}=<p^{\prime}\left(n^{\prime}, \vec{p}_{y}^{\prime}, s^{\prime}\right), n^{\prime}\left(\vec{k}^{\prime}, r^{\prime}\right) \mid T\left\{\int d^{4} x^{\prime} d^{4} x \bar{\psi}_{p}\left(x^{\prime}\right)\left(i g_{\pi N} \gamma^{5}\right) \psi_{p}\left(x^{\prime}\right) \phi_{\pi^{0}}\left(x^{\prime}\right)\right. \\
\left.\bar{\psi}_{n}(x)\left(-i g_{\pi N} \gamma^{5}\right) \psi_{n}(x) \phi_{\pi^{0}}(x)\right\} \mid p\left(n, \vec{p}_{y}, s\right), n(\vec{k}, r)>,
\end{array}
$$

and the other is mediated by the charged pion $S_{c c}$

$$
\begin{array}{r}
S_{c c}=<p^{\prime}\left(n^{\prime}, \vec{p}_{y}^{\prime}, s^{\prime}\right), n^{\prime}\left(\vec{k}^{\prime}, r^{\prime}\right) \mid T\left\{\int d^{4} x^{\prime} d^{4} x \bar{\psi}_{p}\left(x^{\prime}\right)\left(i \sqrt{2} g_{\pi N} \gamma^{5}\right) \psi_{n}\left(x^{\prime}\right) \phi_{\pi^{+}}\left(x^{\prime}\right)\right. \\
\left.\bar{\psi}_{n}(x)\left(i \sqrt{2} g_{\pi N} \gamma^{5}\right) \psi_{p}(x) \phi_{\pi^{-}}(x)\right\} \mid p\left(n, \vec{p}_{y}, s\right), n(\vec{k}, r)>,
\end{array}
$$

where $p(n)$ and $p^{\prime}\left(n^{\prime}\right)$, respectively, correspond to the incoming and outgoing protons (neutrons). Since the neutral pion has no charge its propagator in the external magnetic field does not change, but the propagator of charged pion will be modified. In fact the propagator of the charged pion as a charged scalar particle in an external magnetic field can be obtained as 13

$$
\begin{aligned}
&<T\left\{\phi_{\pi^{+}}\left(x^{\prime}\right) \phi_{\pi^{-}}(x)\right\}>=\exp (-\left.i e \int_{x}^{x^{\prime}} d \xi_{\mu}\left\{A^{\mu}+\frac{1}{2} F^{\mu \nu}(\xi-x)_{\nu}\right\}\right) \int \frac{d^{4} q}{(2 \pi)^{4}} e^{-i q \cdot\left(x^{\prime}-x\right)} \\
& \times \int_{0}^{\infty} \frac{d s}{\cos (e B s)} \exp \left(i s\left\{q_{\|}^{2}-\frac{\tan (e B s)}{e B s} q_{\perp}^{2}-m_{\pi}^{2}\right\}\right),(16
\end{aligned}
$$

in which $F_{\mu \nu}$ is the field strength tensor of the external magnetic field and

$$
q_{\|}^{2}=\left(q_{0}\right)^{2}-\left(q_{3}\right)^{2}, \quad q_{\perp}^{2}=\left(q_{1}\right)^{2}+\left(q_{2}\right)^{2} .
$$

The temperature of a neutron star in a few seconds in the late-time cooling phase, drops to less than $1 \mathrm{MeV}$ therefore the energy is sufficiently low to use the four-fermion approximation, $q^{2} / m_{\pi}^{2}=0$. In this case the propagator (16) can be cast into

$$
\begin{aligned}
<T\left\{\phi_{\pi^{+}}\left(x^{\prime}\right) \phi_{\pi^{-}}(x)\right\}> & =\frac{-i}{m_{\pi}^{2}} D\left(e B / m_{\pi}^{2}\right) \delta^{4}\left(x^{\prime}-x\right) ; \\
D\left(e B / m_{\pi}^{2}\right) & =\int_{0}^{\infty} d s \frac{e^{-s}}{\cosh \left(\frac{e B s}{m_{\pi}^{2}}\right)} .
\end{aligned}
$$

In the four-fermion approximation, $D$ actually shows how the strong coupling constant depends on the external magnetic field. The B-dependence of the coupling constant is explicitly shown in Fig. 1. By using the explicit form of the proton and neutron 


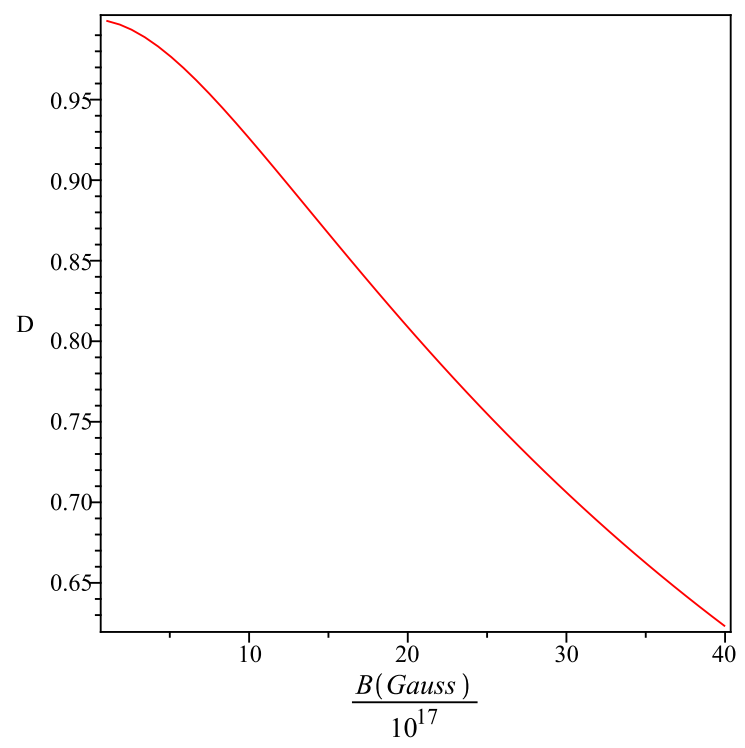

Figure 1: The B-dependence of the squared coupling constant. The variation starts from $10^{15} \mathrm{G}$ but the value decreases significantly from $10^{17} \mathrm{G}$.

field operators (4) and (8) and the definitions of the 1-particle states (17) and (11), the scattering matrix elements can be obtained as follows

$$
S_{n c(c c)}=\frac{1}{L_{x} L_{z} V} N_{p} N_{n} N_{p^{\prime}} N_{n^{\prime}}(2 \pi)^{3} \delta_{y}^{3}\left(p^{\prime}+k^{\prime}-p-k\right)\left(-i \mathcal{M}_{n c(c c)}\right)
$$

where the neutral current scattering amplitude is given by

$$
\mathcal{M}_{n c}=\frac{g_{\pi N}^{2}}{m_{\pi}^{2}} \int d y e^{-i\left(k_{y}^{\prime}-k_{y}\right) y}\left[\bar{U}_{p^{\prime}}\left(y, n^{\prime}, \vec{p}_{y}^{\prime}, s^{\prime}\right) \gamma^{5} U_{p}\left(y, n, \vec{p}_{y}, s\right)\right]\left[\bar{U}_{n^{\prime}}\left(\vec{k}^{\prime}, r^{\prime}\right) \gamma^{5} U_{n}(\vec{k}, r)\right],
$$

and the charged current is

$$
\mathcal{M}_{c c}=2 D \frac{g_{\pi N}^{2}}{m_{\pi}^{2}} \int d y e^{-i\left(k_{y}^{\prime}-k_{y}\right) y}\left[\bar{U}_{p^{\prime}}\left(y, n^{\prime}, \vec{p}_{y}^{\prime}, s^{\prime}\right) \gamma^{5} U_{n}(\vec{k}, r)\right]\left[\bar{U}_{n^{\prime}}\left(\vec{k}^{\prime}, r^{\prime}\right) \gamma^{5} U_{p}\left(y, n, \vec{p}_{y}, s\right)\right] .
$$

Now we are ready to calculate the scattering cross section in the external magnetic field. For this purpose we have

$$
\overline{|S|^{2}}=\frac{1}{\left(2 s_{p}+1\right)} \frac{1}{\left(2 s_{n}+1\right)} \sum_{\text {spin }}\left|S_{n c}+S_{c c}\right|^{2},
$$

where using the normalization relation for the Dirac $\delta$-functions

$$
\left(\delta_{y}^{3}\left(p^{\prime}+k^{\prime}-p-k\right)\right)^{2}=\frac{1}{(2 \pi)^{3}} T L_{x} L_{z} \delta_{y}^{3}\left(p^{\prime}+k^{\prime}-p-k\right),
$$


cast the squared amplitude into

$$
|S|^{2}=\frac{T L_{x} L_{z}}{\left(L_{x} L_{z} V\right)^{2}} N_{p}^{2} N_{n}^{2} N_{p^{\prime}}^{2} N_{n^{\prime}}^{2}(2 \pi)^{3} \delta_{\breve{y}}^{3}\left(p^{\prime}+k^{\prime}-p-k\right)|\mathcal{M}|^{2} .
$$

Now the equations (12), (24) and the deferential phase space of the final states in the external magnetic field

$$
d \rho=\frac{d^{2} p_{y}^{\prime}}{(2 \pi)^{2}} L_{x} L_{z} \frac{d^{3} k^{\prime}}{(2 \pi)^{3}} V,
$$

can be used to find the corresponding deferential cross section as follows

$$
\begin{array}{r}
d \sigma_{B}=\frac{1}{\left|\vec{v}_{p}-\vec{v}_{n}\right|} \frac{1}{\left(2 s_{p}+1\right)\left(2 s_{n}+1\right)} \sum_{\text {spins }} N_{p}^{2} N_{n}^{2} \frac{d^{2} p_{y}^{\prime}}{(2 \pi)^{2}} \frac{d^{3} k^{\prime}}{(2 \pi)^{3}} N_{p^{\prime}}^{2} N_{n^{\prime}}^{2} \\
\times(2 \pi)^{3} \delta_{y}^{3}\left(p^{\prime}+k^{\prime}-p-k\right)|\mathcal{M}|^{2} .
\end{array}
$$

Since the nucleons in the neutron star are nonrelativistic their energies and wave functions can be expanded up to the order of $\mathcal{O}\left(1 / m_{N}\right)$ and the amplitude $\mathcal{M}$ can be obtained analytically as is given in the Appendix B. Meanwhile one can easily show that

$$
N_{p}, N_{n}, N_{p^{\prime}}, N_{n^{\prime}}=1+\mathcal{O}\left(1 / m_{N}^{2}\right)
$$

Therefore the leading term in the scattering cross section (26) is

$$
\begin{aligned}
\sigma_{B} & =\frac{1}{\left|\vec{v}_{p}-\vec{v}_{n}\right|} \sum_{n^{\prime}=0}^{\infty} \int \frac{d^{2} p_{y}^{\prime}}{(2 \pi)^{2}} \frac{d^{3} k^{\prime}}{(2 \pi)^{3}} \\
& \times \frac{1}{\left(2 s_{p}+1\right)\left(2 s_{n}+1\right)} \sum_{\text {spins }}(2 \pi)^{3} \delta_{y^{3}}^{3}\left(p^{\prime}+k^{\prime}-p-k\right)|\mathcal{M}|^{2} .
\end{aligned}
$$

After integrating over $p_{x}^{\prime}, k_{z}^{\prime}$ with the help of the Dirac $\delta$-functions and defining

$$
k_{x}^{\prime}=k_{\perp}^{\prime} \cos \theta^{\prime}, \quad k_{y}^{\prime}=k_{\perp}^{\prime} \sin \theta^{\prime},
$$

equation (28) leads to

$$
\begin{aligned}
\sigma_{B} & =\frac{1}{4 \pi^{2}\left|\vec{v}_{p}-\vec{v}_{n}\right|} \sum_{n^{\prime}=0}^{\infty} \int_{-\infty}^{+\infty} d p_{z}^{\prime} \int_{0}^{2 \pi} d \theta^{\prime} \int_{0}^{+\infty} d k_{\perp}^{\prime} k_{\perp}^{\prime} \\
& \times \frac{1}{\left(2 s_{p}+1\right)\left(2 s_{n}+1\right)} \sum_{\text {spins }} \delta\left(E_{p}^{\prime}+E_{n}^{\prime}-E_{p}-E_{n}\right)|\mathcal{M}|^{2} .
\end{aligned}
$$

By using the nonrelativistic expansion of the energies as are given in the equations (76) and (77) one has

$$
\begin{aligned}
0 & =E_{p}^{\prime}+E_{n}^{\prime}-E_{p}-E_{n} \\
& =\frac{1}{2 m_{N}}\left(k_{\perp}^{\prime 2}+2\left(p_{z}^{\prime}-\frac{1}{2}\left(p_{z}+k_{z}\right)\right)^{2}-2 e B\left(n_{\max }^{\prime}-n^{\prime}\right)\right)
\end{aligned}
$$


where $n_{\text {max }}^{\prime}$ is defined as

$$
n_{\text {max }}^{\prime} \equiv n+\frac{K_{p}}{2}\left(s^{\prime}-s\right)+\frac{K_{n}}{2}\left(r^{\prime}-r\right)+\frac{1}{2 e B}\left(k_{\perp}^{2}+\frac{1}{2}\left(p_{z}-k_{z}\right)^{2}\right) .
$$

The positive solution of (31) is

$$
k_{\perp 0}=\sqrt{2 e B\left(n_{\text {max }}^{\prime}-n^{\prime}\right)-2\left(p_{z}^{\prime}-\frac{1}{2}\left(p_{z}+k_{z}\right)\right)^{2}} .
$$

Now one can perform the integration over $k_{\perp}^{\prime}$ to find

$$
\sigma_{B}=\frac{m_{N}}{4 \pi^{2}\left|\vec{v}_{p}-\vec{v}_{n}\right|} \frac{1}{\left(2 s_{p}+1\right)\left(2 s_{n}+1\right)} \sum_{\text {spins }} \sum_{n^{\prime}=0}^{\mathcal{N}_{\max }^{\prime}} \int_{\mathcal{P}_{z-}^{\prime}}^{\mathcal{P}_{z+}^{\prime}} d p_{z}^{\prime} \int_{0}^{2 \pi} d \theta^{\prime}|\mathcal{M}|^{2},
$$

where

$$
\mathcal{N}_{\text {max }}^{\prime}=\operatorname{INT}\left(n_{\text {max }}^{\prime}\right) \text {, }
$$

while the integration bounds over $p_{z}^{\prime}$ can be determined by the following equation

$$
\mathcal{P}_{z \pm}^{\prime}=\frac{1}{2}\left(p_{z}+k_{z}\right) \pm \sqrt{e B\left(n_{\max }^{\prime}-n^{\prime}\right)}
$$

To evaluate $\sigma_{B}$ in the equation (34) first one needs to determine the maximum Landaulevel that proton can occupy in the initial state, i.e. $\mathcal{N}_{\max }$. Since we would like to consider the process (11) in the temperature range $T \sim 1 \mathrm{Mev}$ [2, [4, [14 therefore nucleons are nonrelativistic and the energy eigenvalues for the protons and neutrons can be given by (76) and (77), respectively. According to the Virial theorem, each nucleon at a temperature $T$ has a momentum for each component about $\left(m_{N} T\right)^{1 / 2}$. Therefore one can easily obtain the maximum Landau-level by comparing the magnetic energy contribution to the proton $n e B / m_{N}$, see (76), with the thermal energy as $\mathcal{N}_{\text {max }}=$ $\operatorname{INT}\left(m_{N} T / e B\right)$.

In this section we obtained the cross-section in the background of a strong magnetic field $\sigma_{B}$ by considering $\mathrm{OPE}$ in the four-fermion approximation. For simplicity we calculate the cross-section in the head-on collisions i.e. $(\vec{p}+\vec{k})_{y}=0$. Since we would like to find if the magnetic field can significantly change the thermal evolution of the neutron stars, it would be better to normalize our result with the cross-section in the absence of the magnetic field, $\sigma_{0}$, but with the same approximations and conditions i.e. $(\vec{p}+\vec{k})=0$, or

$$
\sigma_{0}=\frac{2 g_{\pi N}^{4}}{3 \pi m_{\pi}^{4}\left|\vec{v}_{p}-\vec{v}_{n}\right|} \frac{|\vec{p}|^{5}}{m_{N}^{3}}
$$




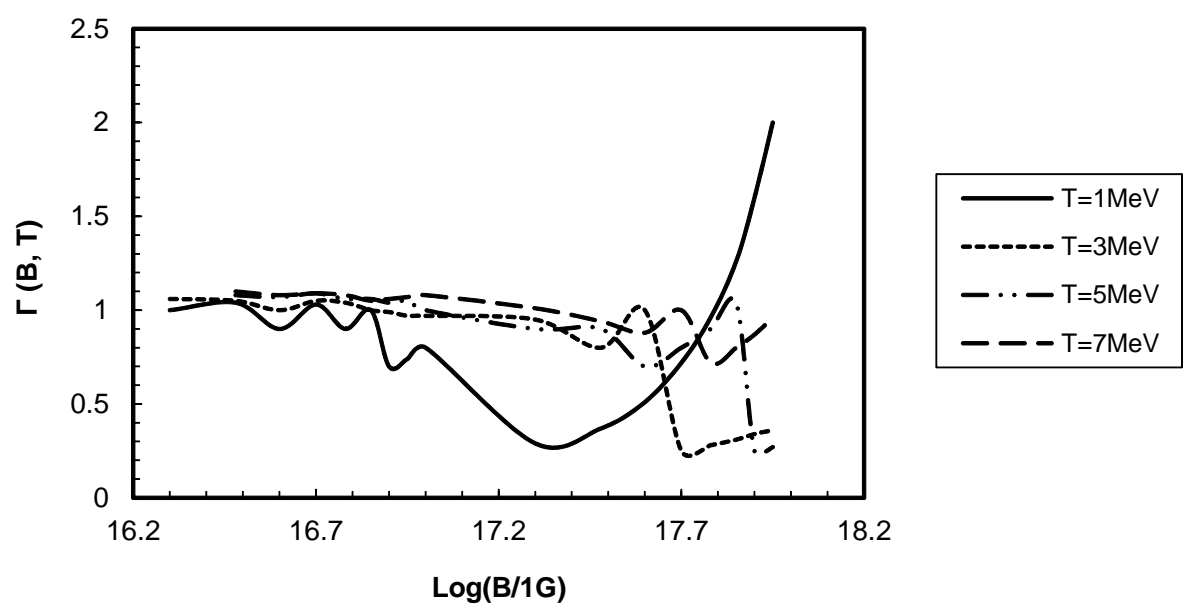

Figure 2: The variation of the $\Gamma$ in terms of $\log (B / 1 \mathrm{G})$ for different temperatures.

Therefore the deviation $\frac{\sigma_{B}}{\sigma_{0}}$ as a function of the magnetic field and temperature can be given by

$$
\begin{aligned}
\Gamma(B, T) & \equiv \frac{1}{\left(1+\mathcal{N}_{\max }\right)} \sum_{n=0}^{\mathcal{N}_{\max }} \frac{\sigma_{B}}{\sigma_{0}}=\frac{3}{8 \pi}\left(\frac{m_{N}}{|\vec{p}|}\right)^{5} \frac{1}{\left(1+\mathcal{N}_{\max }\right)} \sum_{n=0}^{\mathcal{N}_{\max }} \\
& \times \frac{1}{\left(2 s_{p}+1\right)\left(2 s_{n}+1\right)} \sum_{\text {spins }} \sum_{n^{\prime}=0}^{\mathcal{N}_{\max }^{\prime}} \int_{\mathcal{P}_{z-}^{\prime}}^{\mathcal{P}_{z+}^{\prime}} \frac{d p_{z}^{\prime}}{m_{N}} \int_{0}^{2 \pi} d \theta^{\prime} \widetilde{\left.\mathcal{M}\right|^{2}}
\end{aligned}
$$

where

$$
\widetilde{|\mathcal{M}|^{2}}=\frac{m_{\pi}^{4}}{g_{\pi N}^{4}}|\mathcal{M}|^{2} .
$$

The variation of $\Gamma$ as a function of the external magnetic field for different temperatures are shown in the Figures 2 and 3.

\section{Neutrino emissivity}

The NN-scattering cross section in the magnetic fields as large as $10^{15} \mathrm{G}-10^{18} \mathrm{G}$ shows that the strong magnetic filed of the neutron stars might change the neutrino emissivity and therefore the cooling process. The rate of emission and absorbtion of neutrino in the 


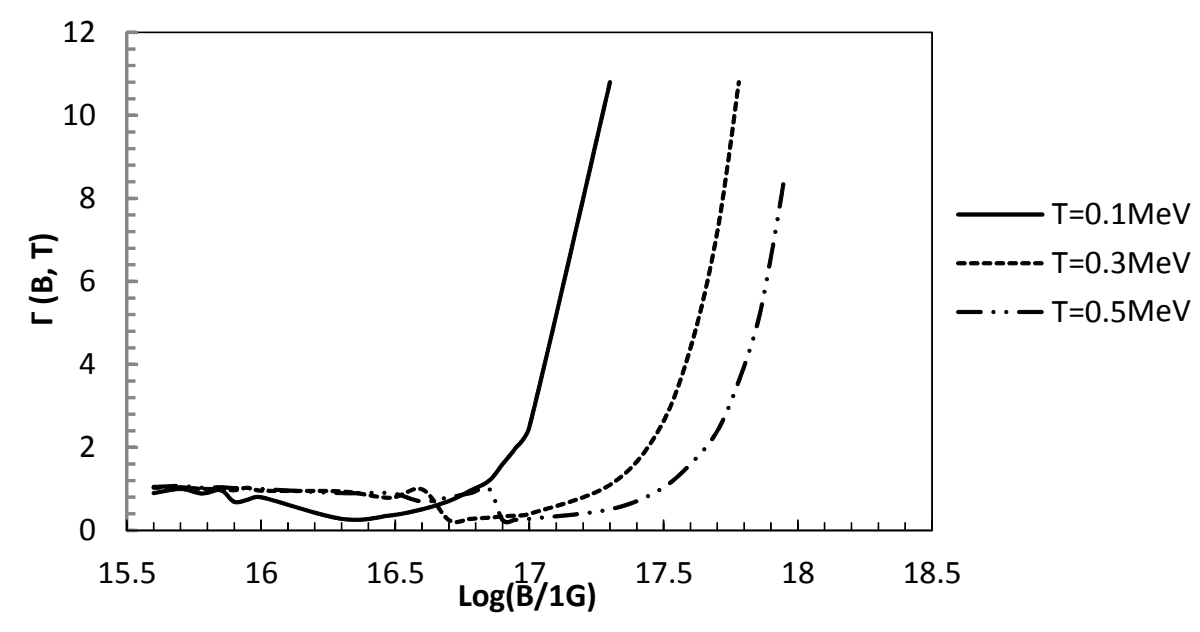

Figure 3: The variation of the $\Gamma$ in terms of $\log (B / 1 \mathrm{G})$ for different temperatures.

strong magnetic field of a neutron star have been already considered for the Urca process in [15], [16] and [17]. In [15] for a strange stars with all charged particles in the lowest Landau level, it is shown that the magnetic field accelerates the cooling process. While in [16] it is found that the direct Urca emissivity is strongly enhanced and oscillates as a function of the magnetic field which globally tends to average out. Here we would like to consider the effect of strong magnetic field on the neutrino bremsstrahlung process (1). To obtain the emissivity at the tree level one should consider all Feynman diagrams at this level. Here for simplicity we just consider the diagram given in Fig, 4 to give an order of magnitude for the emissivity and leave the complete calculation for future study. For this purpose we use the pion-nucleon interaction Lagrangian (13) and the weak interactions Lagrangian

$$
\mathcal{L}_{i n t}=\frac{g}{4 \cos \theta_{\mathrm{w}}}\left[\bar{\psi}_{\nu} \gamma^{\mu}\left(1-\gamma^{5}\right) \psi_{\nu}-\bar{\psi}_{n} \gamma^{\mu}\left(1-\gamma^{5}\right) \psi_{n}+\bar{\psi}_{p} \gamma^{\mu}\left(1-4 \sin ^{2} \theta_{\mathrm{w}}-\gamma^{5}\right) \psi_{p}\right] Z_{\mu}^{0}
$$

According to the Fermi's golden rule, neutrino emissivity in the magnetic field can be written as

$$
\epsilon_{B}=\frac{1}{V T} \sum_{n=0}^{\infty} \sum_{n^{\prime}=0}^{\infty} \int \frac{d^{2} p_{y} L_{x} L_{z}}{(2 \pi)^{2}} \frac{d^{3} k V}{(2 \pi)^{3}} \frac{d^{2} p_{y}^{\prime} L_{x} L_{z}}{(2 \pi)^{2}} \frac{d^{3} k^{\prime} V}{(2 \pi)^{3}} \frac{d^{3} q_{1} V}{(2 \pi)^{3}} \frac{d^{3} q_{2} V}{(2 \pi)^{3}} F\left(\sum_{\text {spins }}|S|^{2}\right) \omega,
$$

where $\omega$ is the total energy of neutrino with the momentum of $q_{1}$ and antineutrino with the momentum of $q_{2}$, all other momenta have the same definition as was given in the 


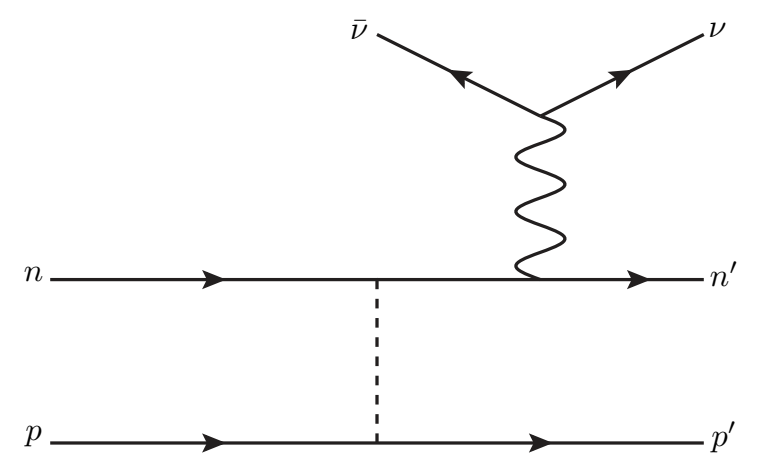

Figure 4: Feynman diagram for neutrino emissivity.

last section. $F$ is defined in terms of the Fermi-Dirac distribution functions of nucleons as

$$
F=f_{p} f_{n}\left(1-f_{p^{\prime}}\right)\left(1-f_{n^{\prime}}\right) ; \quad f=\frac{1}{1+\exp \left(\frac{E-\mu}{T}\right)},
$$

in which $\mu$ is the chemical potential of the nucleon. Now we consider the nucleons, comparing the available energies, as non-relativistic particles. Furthermore the anomalous magnetic moment in the energy spectrum of nucleons are negligible, see Eqs. (76), (177). Meanwhile since the neutrinos are soft the three-momenta of neutrino and antineutrino can be dropped from the Dirac $\delta$-functions. Therefore the spin summed over the squared scattering matrix element can be obtained as

$$
\sum_{\text {spin }}|S|^{2}=\frac{L_{x} L_{z} T}{\left(L_{x} L_{z}\right)^{2} V^{4} 2 E_{1} 2 E_{2}}(2 \pi)^{3} \delta_{y}^{3}\left(p^{\prime}+k^{\prime}+q_{1}+q_{2}-p-k\right) \sum_{\text {spin }}|\mathcal{M}|^{2},
$$

where

$$
\begin{array}{r}
\sum_{\text {spin }}|\mathcal{M}|^{2}=\left(\frac{G_{F} g_{\pi N}^{2}}{\sqrt{2} m_{N}^{2} m_{\pi}^{2}}\right)^{2} \frac{E_{1} E_{2}}{\left(E_{1}+E_{2}\right)^{2}} \vec{k}^{2}\left\{\left(\left(p_{z}^{\prime}-p_{z}\right)^{2}+2 e B\left(n^{\prime}+n\right)\right)\right. \\
\left.\times\left(I_{n, n^{\prime}} I_{n, n^{\prime}}^{*}+I_{n-1, n^{\prime}-1} I_{n-1, n^{\prime}-1}^{*}\right)-4 e B \sqrt{n^{\prime} n}\left(I_{n, n^{\prime}} I_{n-1, n^{\prime}-1}^{*}+I_{n-1, n^{\prime}-1} I_{n, n^{\prime}}^{*}\right)\right\},
\end{array}
$$

in which the functions $I_{n, m}$ are defined in (71). Substituting (43) in the emissivity expression (41) yields

$$
\begin{aligned}
\epsilon_{B}=\sqrt{e B} & \sum_{n=0}^{\infty} \sum_{n^{\prime}=0}^{\infty} \int \frac{d^{2} p_{y}}{(2 \pi)^{2}} \frac{d^{3} k}{(2 \pi)^{3}} \frac{d^{2} p_{y}^{\prime}}{(2 \pi)^{2}} \frac{d^{3} k^{\prime}}{(2 \pi)^{3}} \frac{d^{3} q_{1}}{(2 \pi)^{3}} \frac{1}{2 E_{1}} \frac{d^{3} q_{2}}{(2 \pi)^{3}} \frac{1}{2 E_{2}} \\
& \times(2 \pi)^{3} \delta_{y}^{3}\left(p^{\prime}+k^{\prime}+q_{1}+q_{2}-p-k\right) F\left(\sum_{\text {spins }}|\mathcal{M}|^{2}\right) \omega .
\end{aligned}
$$




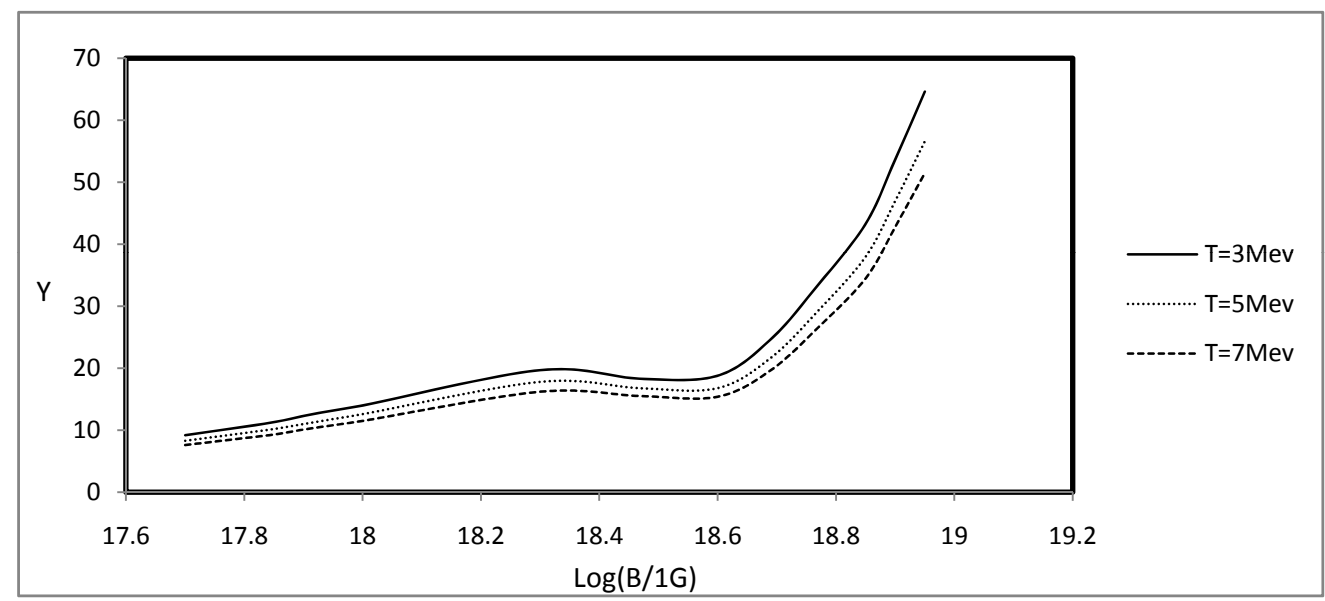

Figure 5: $\Upsilon=\frac{\epsilon_{B}}{\epsilon_{0}}$ is plotted as function of magnetic field for different temperatures.

One should note that the range of $L_{y}$ can be fixed in a natural way as $L_{y}=\frac{1}{\sqrt{e B}}$ [15]. Furthermore to perform the integrals the momentum of particles for a degenerate system, can not be larger than the Fermi momenta that in turns depend on the density of system and implicitly on the temperature [12, so the phase space integrals of the initial particles confined to the Fermi surface. While the remanning integrals on the phase space of the final particles can be accomplished using the momentum conserving Dirac $\delta$-functions. Here we define $\Upsilon=\frac{\epsilon_{B}}{\epsilon_{0}}$ where $\epsilon_{0}$ shows the corresponding emissivity in the absence of magnetic field. The variation of $\Upsilon$ with respect to the neutron star magnetic field for different temperature is shown in Fig 5 .

\section{Conclusion}

We have examined the nucleon-nucleon scattering in a large magnetic background in a neutron star at the temperature about $1 \mathrm{MeV}$. Since the energy is low we can safely use the one-pion-exchange approximation and the four-fermion approximation. In fact the external magnetic field can change the effective coupling constant that is shown in the Fig.1. As the figure shows the coupling is insensitive to the background field up to the $10^{17} \mathrm{G}$ and then by increasing the magnetic field to $4 \times 10^{18} \mathrm{G}$ the squared value of the strong coupling constant decreases to 0.6 its initial value. However the magnetic field 
can change the nucleon wave functions and their corresponding field operators as well, see equations (4) and (8). Therefore the cross-section has an overall change as given in equation (34). The variation of the cross-section, for head-on collisions, in a magnetic background that is normalized with its correspondence without background but with the same approximations are drawn in the Fig.2 and Fig.3 for different temperatures. As the figures show the deviation for the lower temperature start in the lower magnetic fields. Nevertheless to have a significant deviation the magnetic field even for the temperature as low as $0.1 \mathrm{MeV}$, should be grater than $10^{15} \mathrm{G}$. We also studied the neutrino bremsstrahlung emissivity by considering just one Feynman diagram for this process at the tree level to give an estimate on the neutrino emissivity in a strong magnetic field. As is shown in the Fig 5 around $B=10^{18} \mathrm{G}$ the emissivity changes about an order of magnitude with respect to its corresponding value without magnetic filed. Furthermore the enhancement on the emissivity is higher for the lower temperature. Therefore the thermal evolution of the neutron stars in the late time cooling era for $B \geq 10^{15} \mathrm{G}$ can be dramatically changed. 


\section{A Solutions of Dirac equation}

In this Appendix we drive the wave functions of proton and neutron in the external magnetic field by tracing a procedure similar to [18]. Dirac equation for a particle with mass $m$, charge $e=|e|$ and anomalous magnetic moment $\tilde{\mu}=K \frac{e}{2 m}$ in presence of the external magnetic field (2) is

$$
\begin{aligned}
i \frac{\partial \psi}{\partial t} & =H_{D} \psi \\
H_{D} & =\left(\vec{\alpha} . \vec{\Pi}+\beta m-\tilde{\mu} B \beta \Sigma_{z}\right) .
\end{aligned}
$$

Where the canonical momentum $\vec{\Pi}$ is given by the minimal coupling

$$
\vec{\Pi}=-i \vec{\nabla}-e \vec{A}
$$

By using the standard Pauli-Dirac representation for Dirac matrices

$$
\vec{\alpha}=\left(\begin{array}{cc}
0 & \vec{\sigma} \\
\vec{\sigma} & 0
\end{array}\right), \quad \beta=\left(\begin{array}{cc}
I & 0 \\
0 & -I
\end{array}\right), \quad \Sigma_{z}=\left(\begin{array}{cc}
\sigma_{z} & 0 \\
0 & \sigma_{z}
\end{array}\right), \quad \gamma^{5}=\left(\begin{array}{cc}
0 & I \\
I & 0
\end{array}\right),
$$

and general form of the wave function

$$
\psi(t, \vec{x})=e^{-i p \cdot x_{y}}\left(\begin{array}{l}
u_{1} \\
u_{2} \\
u_{3} \\
u_{4}
\end{array}\right),
$$

the following system of equations will be obtained

$$
\begin{aligned}
(E-m+\tilde{\mu} B) u_{1}-p_{z} u_{3}-\left(\Pi_{x}-i \Pi_{y}\right) u_{4} & =0 \\
(E-m-\tilde{\mu} B) u_{2}+p_{z} u_{4}-\left(\Pi_{x}+i \Pi_{y}\right) u_{3} & =0 \\
(E+m-\tilde{\mu} B) u_{3}-p_{z} u_{1}-\left(\Pi_{x}-i \Pi_{y}\right) u_{2} & =0 \\
(E+m+\tilde{\mu} B) u_{4}+p_{z} u_{2}-\left(\Pi_{x}+i \Pi_{y}\right) u_{1} & =0 .
\end{aligned}
$$

To solve equations (50) one can assume

$$
u_{1}=C_{1} I_{n}(\xi), \quad u_{2}=C_{2} I_{n-1}(\xi), \quad u_{3}=C_{3} I_{n}(\xi), \quad u_{4}=C_{4} I_{n-1}(\xi)
$$

where the function $I_{n}(\xi)$ is defined as

$$
\begin{array}{rlrl}
I_{n}(\xi) & \equiv N_{n} e^{-\xi^{2} / 2} H_{n}(\xi) ; & n=0,1,2, \ldots \\
\xi & \equiv \sqrt{e B} y+\frac{p_{x}}{\sqrt{e B}}, \quad N_{n}=\sqrt{\frac{\sqrt{e B}}{\sqrt{\pi} 2^{n} n !}} .
\end{array}
$$


The function $H_{n}(\xi)$ is the Hermit polynomial and in according to the definition $H_{-1}(\xi)=$ 0 . Using this choice for normalization constant $N_{n}$, it can be showed that the functions $I_{n}(\xi)$ satisfy the completeness relation

$$
\sum_{n=0}^{\infty} I_{n}(\xi) I_{n}\left(\xi^{\prime}\right)=\delta\left(y-y^{\prime}\right),
$$

where $\xi^{\prime}$ is given by replacing $y$ by $y^{\prime}$ in the definition of the $\xi$ (52). After substituting equations (51) in the system of equations (50), the system of linear equations for coefficients $C_{i}$ will be obtained as follows

$$
\begin{aligned}
& (E \mp m \pm \tilde{\mu} B) C_{1,3}-p_{z} C_{3,1}-\sqrt{2 n e B} C_{4,2}=0 \\
& (E \mp m \mp \tilde{\mu} B) C_{2,4}+p_{z} C_{4,2}-\sqrt{2 n e B} C_{3,1}=0
\end{aligned}
$$

Equations (54) have a non trivial solutions for $C_{i}$ if the Determinant of the coefficients be zero. Imposing this condition yields the spectrum of the energy

$$
E_{n, s}=\sqrt{p_{z}^{2}+\left(\sqrt{m^{2}+2 n e B}-s \tilde{\mu} B\right)^{2}} ; \quad s= \pm 1 .
$$

In the energy spectrum (55) index of $n$ is called Landau level. To solve equations (54) it is better to introduce the fermion spin projection operator on the direction of the magnetic field as

$$
\hat{\mu}_{z}=m \Sigma_{z}+i \gamma^{5} \beta(\vec{\Sigma} \times \vec{\Pi})_{z}
$$

Since the operator $\hat{\mu}_{z}$ commutes with the Dirac Hamiltonian $H_{D}$, the wave functions are eigenstate of this operator

$$
\hat{\mu}_{z} \psi=\mu_{0} \psi
$$

where $\mu_{0}$ is the eigenvalue of the operator $\hat{\mu}_{z}$. Then using the equations (57) and (51), one leads to the following system of liner equations for the coefficients $C_{i}$

$$
\left(m \mp \mu_{0}\right) C_{1,4} \pm \sqrt{2 n e B} C_{4,1}=0, \quad\left(m \pm \mu_{0}\right) C_{2,3} \pm \sqrt{2 n e B} C_{3,2}=0 .
$$

Again by imposing the condition that, Determinant of the coefficients must be zero, $\mu_{0}$ can be determined as

$$
\mu_{0}=s \sqrt{m^{2}+2 n e B} ; \quad s= \pm 1 .
$$

Now by using the equations (54), (58) and the following normalization condition, the coefficients $C_{i}$ can be determined

$$
\sum_{i=1}^{i=4}\left|C_{i}\right|^{2}=1
$$


Therefore the wave function can be obtained as

$$
\begin{aligned}
\psi_{s}(x) & =N_{s} e^{-i p \cdot x_{y}} U_{s}\left(y, n, \vec{p}_{y}\right) ; \\
U_{s}\left(y, n, \vec{p}_{y}\right) & =\left(\begin{array}{c}
\left(\frac{1+s}{2}+\frac{1-s}{2} \tilde{p}_{z s} \tilde{\gamma}_{n}\right) I_{n}(\xi) \\
\left(-\frac{1+s}{2} \tilde{p}_{z s} \tilde{\gamma}_{n}+\frac{1-s}{2}\right) I_{n-1}(\xi) \\
\left(\frac{1+s}{2} \tilde{p}_{z s}+\frac{1-s}{2} \tilde{\gamma}_{n}\right) I_{n}(\xi) \\
\left(\frac{1+s}{2} \tilde{\gamma}_{n}-\frac{1-s}{2} \tilde{p}_{z s}\right) I_{n-1}(\xi)
\end{array}\right), \quad s= \pm 1,
\end{aligned}
$$

where the quantities $\tilde{p}_{z s}$ and $\tilde{\gamma}_{n}$ are defined as

$$
\tilde{p}_{z s}=\frac{p_{z}}{E_{n, s}+\sqrt{E_{n, s}^{2}-p_{z}^{2}}}, \quad \quad \tilde{\gamma}_{n}=\frac{\sqrt{2 n e B}}{m+\sqrt{m^{2}+2 n e B}},
$$

and the normalization constant $N_{s}$ of the wave function is

$$
N_{s}=\frac{1}{\sqrt{\left(1+\tilde{p}_{z s}^{2}\right)\left(1+\tilde{\gamma}_{n}^{2}\right)}} .
$$

One can easily find the wave functions for the negative energy spinors by doing the similar procedure. In this case it is easier to determine the upper component with respect to the lower one. After some manipulations one has

$$
\begin{aligned}
\psi_{s}(x) & =N_{s} e^{i p . x_{y}} V_{s}\left(y, n, \vec{p}_{y}\right) ; \\
V_{s}\left(y, n, \vec{p}_{y}\right) & =\left(\begin{array}{c}
\left(\frac{1+s}{2} \tilde{p}_{z s}-\frac{1-s}{2} \tilde{\gamma}_{n}\right) I_{n}(\tilde{\xi}) \\
\left(-\frac{1+s}{2} \tilde{\gamma}_{n}-\frac{1-s}{2} \tilde{p}_{z s}\right) I_{n-1}(\tilde{\xi}) \\
\left(\frac{1+s}{2}-\frac{1-s}{2} \tilde{p}_{z s} \tilde{\gamma}_{n}\right) I_{n}(\tilde{\xi}) \\
\left(\frac{1+s}{2} \tilde{p}_{z s} \tilde{\gamma}_{n}+\frac{1-s}{2}\right) I_{n-1}(\tilde{\xi})
\end{array}\right) ; \quad \tilde{\xi} \equiv \sqrt{e B} y-\frac{p_{x}}{\sqrt{e B}},
\end{aligned}
$$

where quantities $\tilde{p}_{z s}$ and $\tilde{\gamma}_{n}$ and normalization constant $N_{s}$ have the same definition as the positive energy solutions and can be determined, respectively, from equations (62) and (63). Neutron has not charge but due to its anomalous magnetic moment its wave function and energy spectrum can be modified by the magnetic field. Solving Dirac equation for this case is simpler than proton case. By doing the same procedure as the proton case, the spectrum can be obtained as

$$
E_{s}=\sqrt{p_{z}^{2}+\left(\sqrt{m^{2}+p_{\perp}^{2}}-s \tilde{\mu} B\right)^{2}} ; \quad s= \pm 1,
$$

and the wave functions will be given by

$$
\begin{aligned}
& \psi_{s}(x)= N_{s} e^{-i p \cdot x} U_{s}(\vec{p}) ; \\
& U_{s}(\vec{p})=\left(\begin{array}{c}
\left(\frac{1+s}{2}+\frac{1-s}{2} \tilde{p}_{z s} \tilde{p}_{s}\right) \\
\left(-\frac{1+s}{2} \tilde{p}_{z s} \tilde{p}_{s}+\frac{1-s}{2}\right) \\
\left(\frac{1+s}{2} \tilde{p}_{z s}+\frac{1-s}{2} \tilde{p}_{s}\right) \\
\left(\frac{1+s}{2} \tilde{p}_{s}-\frac{1-s}{2} \tilde{p}_{z s}\right)
\end{array}\right), \quad s= \pm 1,
\end{aligned}
$$


where quantities $\tilde{p}_{z s}, \tilde{p}_{s}$ and $p_{\perp}$ are defined by

$$
\begin{aligned}
\tilde{p}_{z s} & =\frac{p_{z}}{E_{s}+\sqrt{E_{s}^{2}-p_{z}^{2}}}, & \tilde{p}_{s} & =\frac{p_{s}}{m+\sqrt{m^{2}+p_{\perp}^{2}}} ; \\
p_{s} & =p_{x}+\operatorname{sip}_{y}, & p_{\perp} & =\sqrt{p_{+} p_{-}}
\end{aligned}
$$

and the normalization constant given by

$$
N_{s}=\frac{1}{\sqrt{\left(1+\tilde{p}_{z s}^{2}\right)\left(1+\tilde{p}_{\perp}^{2}\right)}} ; \quad \quad \tilde{p}_{\perp}=\sqrt{\tilde{p}_{+} \tilde{p}_{-}} .
$$

Finally the solutions for the negative energy case can be obtained as

$$
\begin{aligned}
\psi_{s}(x)= & N_{s} e^{i p . x} V_{s}(\vec{p}) \\
V_{s}(\vec{p})= & \left(\begin{array}{c}
\left(\frac{1+s}{2} \tilde{p}_{z s}+\frac{1-s}{2} \tilde{p}_{s}\right) \\
\left(\frac{1+s}{2} \tilde{p}_{s}-\frac{1-s}{2} \tilde{p}_{z s}\right) \\
\left(\frac{1+s}{2}+\frac{1-s}{2} \tilde{p}_{z s} \tilde{p}_{s}\right) \\
\left(-\frac{1+s}{2} \tilde{p}_{z s} \tilde{p}_{s}+\frac{1-s}{2}\right)
\end{array}\right) .
\end{aligned}
$$

Definition of the parameters in the wave functions (69) are the same as the equations (67) and (68).

\section{B Average of the squared scattering matrix ele- ments}

The matrix elements that are sum of the neutral (20) and the charge current amplitude (21), contain the following integrals

$$
I_{n, m}=\int d y e^{-i\left(k_{y}^{\prime}-k_{y}\right) y} I_{n}(\xi) I_{m}\left(\xi_{*}\right),
$$

where $\xi_{*}$ is given by replacing $p_{x}$ by $p_{x}^{\prime}$ in the definition of the $\xi$ (52). Details of this integration can be found in [19], however the final answer is as follows

$$
\begin{array}{ll}
I_{n, m}=\sqrt{\frac{n !}{m !}} e^{-\eta^{2} / 2} e^{i \phi_{0}}\left(\eta_{x}+i \eta_{y}\right)^{m-n} L_{n}^{m-n}\left(\eta^{2}\right) ; \quad m \geq n \geq 0, \\
I_{n, m}=\sqrt{\frac{m !}{n !}} e^{-\eta^{2} / 2} e^{i \phi_{0}}\left(-\eta_{x}+i \eta_{y}\right)^{n-m} L_{m}^{n-m}\left(\eta^{2}\right) ; & n \geq m \geq 0,
\end{array}
$$

in which $L_{n}^{k}$ is the generalized Laguerre polynomial and variables $\eta$ and $\phi_{0}$ are defined as

$$
\begin{aligned}
\eta_{x}=\frac{p_{x}^{\prime}-p_{x}}{\sqrt{2 e B}}, & \eta_{y}=-\frac{k_{y}^{\prime}-k_{y}}{\sqrt{2 e B}} \\
\eta^{2}=\eta_{x}^{2}+\eta_{y}^{2}, & \phi_{0}=\frac{\left(p_{x}^{\prime}+p_{x}\right)\left(k_{y}^{\prime}-k_{y}\right)}{2 e B} .
\end{aligned}
$$


By using the explicit form of the wave functions and the definition (70), one can find the nonrelativistic leading order term in the amplitude as follows

$$
\mathcal{M}=\mathcal{M}_{n c}+\mathcal{M}_{c c}
$$

such that the neutral current amplitude matrix element is given by

$$
\begin{aligned}
\mathcal{M}_{n c} & =\frac{g_{\pi N}^{2}}{m_{\pi}^{2}}\left[\left(\frac{1+r}{2}\right)\left(\frac{1+r^{\prime}}{2}\right)\left(\tilde{k}_{z}^{\prime}-\tilde{k}_{z}\right)+\left(\frac{1+r}{2}\right)\left(\frac{1-r^{\prime}}{2}\right)\left(\tilde{k}_{+}^{\prime}-\tilde{k}_{+}\right)\right. \\
& \left.+\left(\frac{1-r}{2}\right)\left(\frac{1+r^{\prime}}{2}\right)\left(\tilde{k}_{-}^{\prime}-\tilde{k}_{-}\right)-\left(\frac{1-r}{2}\right)\left(\frac{1-r^{\prime}}{2}\right)\left(\tilde{k}_{z}^{\prime}-\tilde{k}_{z}\right)\right] \\
& \times\left[\left\{\left(\frac{1+s}{2}\right)\left(\frac{1+s^{\prime}}{2}\right)\left(\tilde{p}_{z}^{\prime}-\tilde{p}_{z}\right)+\left(\frac{1+s}{2}\right)\left(\frac{1-s^{\prime}}{2}\right) \tilde{\gamma}_{n^{\prime}}-\left(\frac{1-s}{2}\right)\left(\frac{1+s^{\prime}}{2}\right) \tilde{\gamma}_{n}\right\} I_{n, n^{\prime}}\right. \\
& \left.-\left\{\left(\frac{1-s}{2}\right)\left(\frac{1-s^{\prime}}{2}\right)\left(\tilde{p}_{z}^{\prime}-\tilde{p}_{z}\right)-\left(\frac{1-s}{2}\right)\left(\frac{1+s^{\prime}}{2}\right) \tilde{\gamma}_{n^{\prime}}+\left(\frac{1+s}{2}\right)\left(\frac{1-s^{\prime}}{2}\right) \tilde{\gamma}_{n}\right\} I_{n-1, n^{\prime}-1}\right],
\end{aligned}
$$

and the charged current amplitude is given by

$$
\begin{aligned}
\mathcal{M}_{c c} & =\frac{2 D g_{\pi N}^{2}}{m_{\pi}^{2}}\left[\left\{\left(\frac{1+r}{2}\right)\left(\frac{1+s^{\prime}}{2}\right)\left(\tilde{p}_{z}^{\prime}-\tilde{k}_{z}\right)+\left(\frac{1+r}{2}\right)\left(\frac{1-s^{\prime}}{2}\right) \tilde{\gamma}_{n^{\prime}}-\left(\frac{1-r}{2}\right)\left(\frac{1+s^{\prime}}{2}\right) \tilde{k}_{-}\right\}\right. \\
& \times\left\{\left(\frac{1+s}{2}\right)\left(\frac{1+r^{\prime}}{2}\right)\left(\tilde{k}_{z}^{\prime}-\tilde{p}_{z}\right)+\left(\frac{1+s}{2}\right)\left(\frac{1-r^{\prime}}{2}\right) \tilde{k}_{+}^{\prime}-\left(\frac{1-s}{2}\right)\left(\frac{1+r^{\prime}}{2}\right) \tilde{\gamma}_{n}\right\} I_{n, n^{\prime}} \\
& +\left\{-\left(\frac{1+r}{2}\right)\left(\frac{1-s^{\prime}}{2}\right) \tilde{k}_{+}+\left(\frac{1-r}{2}\right)\left(\frac{1+s^{\prime}}{2}\right) \tilde{\gamma}_{n^{\prime}}-\left(\frac{1-r}{2}\right)\left(\frac{1-s^{\prime}}{2}\right)\left(\tilde{p}_{z}^{\prime}-\tilde{k}_{z}\right)\right\} \\
& \times\left\{\left(\frac{1+s}{2}\right)\left(\frac{1+r^{\prime}}{2}\right)\left(\tilde{k}_{z}^{\prime}-\tilde{p}_{z}\right)+\left(\frac{1+s}{2}\right)\left(\frac{1-r^{\prime}}{2}\right) \tilde{k}_{+}^{\prime}-\left(\frac{1-s}{2}\right)\left(\frac{1+r^{\prime}}{2}\right) \tilde{\gamma}_{n}\right\} I_{n, n^{\prime}-1} \\
& +\left\{\left(\frac{1+r}{2}\right)\left(\frac{1+s^{\prime}}{2}\right)\left(\tilde{p}_{z}^{\prime}-\tilde{k}_{z}\right)+\left(\frac{1+r}{2}\right)\left(\frac{1-s^{\prime}}{2}\right) \tilde{\gamma}_{n^{\prime}}-\left(\frac{1-r}{2}\right)\left(\frac{1+s^{\prime}}{2}\right) \tilde{k}_{-}\right\} \\
& \times\left\{-\left(\frac{1+s}{2}\right)\left(\frac{1-r^{\prime}}{2}\right) \tilde{\gamma}_{n}+\left(\frac{1-s}{2}\right)\left(\frac{1+r^{\prime}}{2}\right) \tilde{k}_{-}^{\prime}-\left(\frac{1-s}{2}\right)\left(\frac{1-r^{\prime}}{2}\right)\left(\tilde{k}_{z}^{\prime}-\tilde{p}_{z}\right)\right\} I_{n-1, n^{\prime}} \\
& +\left\{\left(\frac{1+r}{2}\right)\left(\frac{1-s^{\prime}}{2}\right) \tilde{k}_{+}-\left(\frac{1-r}{2}\right)\left(\frac{1+s^{\prime}}{2}\right) \tilde{\gamma}_{n^{\prime}}+\left(\frac{1-r}{2}\right)\left(\frac{1-s^{\prime}}{2}\right)\left(\tilde{p}_{z}^{\prime}-\tilde{k}_{z}\right)\right\} \\
& \left.\times\left\{\left(\frac{1+s}{2}\right)\left(\frac{1-r^{\prime}}{2}\right) \tilde{\gamma}_{n}-\left(\frac{1-s}{2}\right)\left(\frac{1+r^{\prime}}{2}\right) \tilde{k}_{-}^{\prime}+\left(\frac{1-s}{2}\right)\left(\frac{1-r^{\prime}}{2}\right)\left(\tilde{k}_{z}^{\prime}-\tilde{p}_{z}\right)\right\} I_{n-1, n^{\prime}-1}\right] .
\end{aligned}
$$

Using the following nonrelativistic expansion of the energy for proton as

$$
E_{n, s}=m_{N}+\frac{p_{z}^{2}}{2 m_{N}}+\frac{n e B}{m_{N}}-s \tilde{\mu}_{p} B
$$

and for the neutron as

$$
E_{s}=m_{N}+\frac{\vec{p}^{2}}{2 m_{N}}-s \tilde{\mu}_{n} B
$$


along with the equations (62) and (67) lead to

$$
\tilde{p}_{z}=\frac{p_{z}}{2 m_{N}}, \quad \tilde{\gamma}_{n}=\frac{\sqrt{2 n e B}}{2 m_{N}}, \quad \tilde{k}_{z}=\frac{k_{z}}{2 m_{N}}, \quad \tilde{k}_{ \pm}=\frac{k_{ \pm}}{2 m_{N}},
$$

with the similar definitions for the primed quantities. 


\section{References}

[1] F. Haberl, Astrophys. and Space Sci. J. 308, 181 (2007).

[2] S. Tsuruta, Phys. Rep. 292, 1 (1998).

[3] C. Thompson and R. C. Duncan, Astrophys. J. 408, 194 (1993).

[4] R. D. Blandford, J. H. Applegate and L. Hernquist, Mon. Not. Roy. Astr. Soc. 204, 1025 (1983).

[5] R. C. Duncan and C. Thompson, Astrophys. J. 392, L9 (1992); C. Thompson and R. C. Duncan, Astrophys. J. 473, 322 (1996); C. Kouveliotou et al., Nature 393, 235 (1998).

[6] S. Mereghetti, Astron. Astrophys. Rev. 15, 225 (2008) [arXiv:0804.0250 [astro-ph]].

[7] R. N. Manchester and J. H. Taylor, Astron. J.86, 1953 (1981).

[8] D. Lai, Rev. Mod. Phys. 73, 629 (2001) arXiv:astro-ph/0009333.

[9] N. Itoh, Mon. Not. Roy. Astr. Soc. 173, 1 (1975).

[10] Deborah N. Aguilera, Jose A. Pons and Juan A. Miralles, Astron. Astrophys. J. 486, 255 (2008).

[11] C. Hanhart, D. R. Phillips and S. Reddy, Phys. Lett. B 499, 9 (2001); C. Hanhart, D. R. Phillips, S. Reddy and M. J. Savage, Nucl. Phys. B 595, 335 (2001).

[12] B. L. Friman and O. V. Maxwell, Astrophys. J. 232, 541 (1979).

[13] A. Erdas and G. Feldman, Nucl. Phys. B 343, 597 (1990).

[14] W. Keil, H. T. Janka and G. Raffelt, Phys. Rev. D 51, 6635 (1995) arXiv:hep-ph/9410229|; S. Hannestad and G. Raffelt, Astrophys. J. 507, 339 (1998) [arXiv:astro-ph/9711132].

[15] X. W. Liu, X. P. Zheng and D. F. Hou, Astropart. Phys. 24, 92 (2005) arXiv:astro-ph/0412515.

[16] Mario Riquelme, Andreas Reisenegger, Olivier Espinosa and Claudio O. Dib, Astrophys. J. 439, 427 (2005). 
[17] Huaiyu Duan and Yong-Zhong Qian, Phys. Rev. D 72, 023005 (2005).

[18] I. Mamsourov and H. Goudarzi, [arXiv:hep-ph/0404086].

[19] D. A. Dicus, W. W. Repko and T. M. Tinsley, Phys. Rev. D 76, 025005 (2007) [Erratum-ibid. D 76, 089903 (2007)] [arXiv:0704.1695 [hep-ph]]. 